\title{
Manobras de desobstrução e acesso as vias aéreas nas emergências odontológicas
}

\author{
Airway management in dental emergency situations \\ Manejo de la vía aérea em situaciones de emergência odontológicas
}

Lohana Maylane Aquino Correia de Lima ORCID: https://orcid.org/0000-0002-1864-8329

Universidade Federal de Pernambuco, Brasil E-mail:Lohanawatson@hotmail.com

Mayana Aquino Correia de Lima ORCID: https://orcid.org/0000-0002-6419-2924

Universidade Católica de Pernambuco, Brasil E-mail: mayana.2019230032@unicap.br

Rayana Cruz Correia de Lima

ORCID: https://orcid.org/0000-0002-9613-3363 Universidade de Pernambuco, Brasil E-mail: rayanalm@gmail.com

Milena Mello Varela Ayres de Melo Pinheiro ORCID: https://orcid.org/0000-0002-5956-1553

Faculdade de Medicina de Olinda, Brasil E-mail: milena_varela@hotmail.com

Rodrigo Henrique Mello Varela Ayres de Melo ORCID: https://orcid.org/0000-0003-4076-5035 Sociedade Sulina - Hospital Divina Providência, Brasil E-mail: rodrigoayres@msn.com

Deise Louise Bohn Rhoden ORCID: https://orcid.org/0000-0003-4675-5137 Universidade Luterana do Brasil, Brasil

E-mail: deiserhoden17@gmail.com

Victor Leonardo Mello Varela Ayres de Melo ORCID: https://orcid.org/0000-0003-3758-6060

Universidade Federal de Pernambuco, Brasil E-mail: victorlmvamelo@gmail.com

Frederico Marcio Varela Ayres de Melo Junior ORCID: https://orcid.org/0000-0001-7377-2392

Universidade Maurício de Nassau, Brasil E-mail: fmvamj31@hotmail.com

Bruna Heloísa Costa Varela Ayres de Melo ORCID: https://orcid.org/0000-0002-3158-5131 Centro Universitário Facex, Brasil E-mail: bvmelo98@gmail.com Júlia de Souza Beck ORCID: https://orcid.org/0000-0002-3647-7367 Universidade Maurício de Nassau, Brasil E-mail: juliabeck@hotmail.com Maria Luísa Alves Lins

ORCID: https://orcid.org/0000-0002-3957-6117

Universidade Federal de Pernambuco, Brasil E-mail: linsluisam@gmail.com

Jussara Diana Varela Ayres de Melo ORCID: https://orcid.org/0000-0003-0759-6188 Faculdade de Comunicação e Turismo de Olinda, Brasil E-mail: Jussara_varela@hotmail.com

Nely Dulce Varela de Melo Costa Freitas ORCID: https://orcid.org/0000-0002-2101-5143

Universidade Maurício de Nassau, Brasil E-mail: nelyvarela@hotmail.com Neme Portal Bustamante ORCID: https://orcid.org/0000-0002-5061-9421 Universidad Nacional Federico Villarreal, Peru E-mail: portal_de_los_implantes@yahoo.com

Ricardo Eugenio Varela Ayres de Melo ORCID: https://orcid.org/0000-0003-1401-457X

Universidade Federal de Pernambuco, Brasil E-mail:revamelo@hotmail.com 


\begin{abstract}
Resumo
A via aérea humana é um sistema composto de importantes estruturas anatômicas que protegem e mantêm a permeabilidade. Este trabalho objetiva apresentar as manobras de desobstrução e acesso às vias aéreas em situações de emergência. Realizou-se uma revisão de literatura com busca nas bases de dados Scielo e Embase. Para isto, utilizouse descritores "Airway Obstruction" e "Emergency" e "Dentistry" com 40 artigos triados. Nas manobras básicas, a manobra de Heimlich consiste na compressão infradiafragmática em "L". A manobra de Jaw Thrust é caracterizada pela protrusão da mandíbula, enquanto que Chin Lift realiza-se a hiperextensão cervical e elevação mentual. Nas manobras avançadas a Intubação Endotraqueal garante o fluxo do ar nas vias aéreas por meio da aposição de uma cânula por via oral ou nasal, a Cricotireoidostomia é um procedimento invasivo que cria uma abertura através da membrana cricotireoidea, enquanto que a Traqueostomia consiste em um procedimento cirúrgico para a criação de uma abertura entre o $2^{0}$ e o $3^{0}$ anel traqueal. Por isso, a manobra de Heimlich é eleita como primeira alternativa nas situações de obstrução aguda seguida das manobras de Jaw Thrust, Chin Lift e a Intubação Endotraqueal. A Cricotireoidostomia é o procedimento invasivo de primeira escolha para o reestabelecimento da ventilação em situações de emergência, enquanto que a Traqueostomia, de caráter eletivo, demanda maior tempo de procedimento. Portanto, a obstrução aguda das vias aéreas superiores é uma complicação grave e de caráter emergencial, por isso deve o CirurgiãoDentista estar preparado para reverter esse quadro rapidamente nessas situações.

Palavras-chave: Manuseio das vias aéreas; Emergências; Odontologia.
\end{abstract}

\begin{abstract}
The human airway is a system composed of important anatomical structures that protect and maintain permeability. This paper aims to present airway clearance and access maneuvers in emergency situations. A literature review was carried out by searching the Scielo and Embase databases. For this, we used descriptors "Airway Obstruction" and "Emergency" and "Dentistry" with 40 articles screened. In basic maneuvers, the Heimlich maneuver consists of infradiaphragmatic compression in an " $\mathrm{"} \mathrm{shape.} \mathrm{The} \mathrm{Jaw} \mathrm{Thrust} \mathrm{maneuver} \mathrm{is} \mathrm{characterized} \mathrm{by} \mathrm{the} \mathrm{protrusion} \mathrm{of} \mathrm{the}$ jaw, while the Chin Lift involves cervical hyperextension and mental elevation. In advanced maneuvers, Endotracheal Intubation ensures airflow in the airways through the placement of an oral or nasal cannula. Cricothyroidotomy is an invasive procedure that creates an opening through the cricothyroid membrane, while the Tracheostomy is a surgical procedure to create an opening between the 2 nd and 3 rd tracheal ring. Therefore, the Heimlich maneuver is chosen as the first alternative in situations of acute obstruction, followed by the Jaw Thrust, Chin Lift and Endotracheal Intubation maneuvers. Cricothyroidotomy is the invasive procedure of first choice for reestablishing ventilation in emergency situations, while tracheostomy, which is elective, requires longer procedure time. Therefore, the acute obstruction of the upper airways is a serious and emergency complication, so the dentist must be prepared to quickly reverse this condition in these situations.
\end{abstract}

Keywords: Airway management; Emergencies; Dentistry.

\title{
Resumen
}

La vía aérea humana es un sistema compuesto por importantes estructuras anatómicas que protegen y mantienen la permeabilidad. Este artículo tiene como objetivo presentar las maniobras de despeje y acceso de las vías respiratorias en situaciones de emergencia. Se realizó una revisión de la literatura mediante búsquedas en las bases de datos Scielo y Embase. Para ello, se utilizaron los descriptores "Obstrucción de la vía aérea" y "Emergencia" y "Odontología" con 40 artículos seleccionados. En las maniobras básicas, la maniobra de Heimlich consiste en una compresión infradiafragmática en forma de "L". La maniobra de empuje de la mandíbula se caracteriza por la protrusión de la mandíbula, mientras que el levantamiento de mentón implica hiperextensión cervical y elevación mental. En maniobras avanzadas, la intubación endotraqueal asegura el flujo de aire en las vías respiratorias mediante la colocación de una cánula oral o nasal. La cricotiroidotomía es un procedimiento invasivo que crea una abertura a través de la membrana cricotiroidea, mientras que la traqueotomía consiste en un procedimiento quirúrgico para crear una abertura entre el segundo y tercer anillo traqueal. Por tanto, se elige la maniobra de Heimlich como primera alternativa en situaciones de obstrucción aguda, seguida de las maniobras de Empuje de la mandíbula, Levantamiento de mentón e Intubación endotraqueal. La cricotiroidotomía es el procedimiento invasivo de primera elección para restablecer la ventilación en situaciones de emergencia, mientras que la traqueotomía, que es electiva, requiere un tiempo de procedimiento más prolongado. Por lo tanto, la obstrucción aguda de las vías respiratorias superiores es una complicación grave y de emergencia, por lo que el odontólogo debe estar preparado para revertir rápidamente esta condición en estas situaciones.

Palabras clave: Manejo de la vía aérea; Urgencias Médicas; Odontología.

\section{Introdução}

O estabelecimento precoce de uma via aérea patente e segura é um princípio básico e imprescindível no suporte a vida. Para um adequado acesso às vias aéreas é necessário entender às condições do paciente, o cenário clínico no qual ele se encontra e quais as habilidades do profissional que irá atende-lo (Macêdo et al., 2016). A obstrução das vias aéreas pode ser 
apresentada de duas formas, quando compromete às vias aéreas superiores se estendedo nariz ou da boca até a laringe enquanto que a obstrução das vias aéreas inferiores estar relacionada à árvore traqueobrônquica (Lynch \& Crawley, 2018). Por isso, todo Cirurgião-Dentista tem que saber que a obstrução das vias aéreas é uma emergência que ameaça a vida e requer do profissional um reconhecimento e gerenciamento imediato (Nama et al., 2020).

No cenário pré-hospitalar, os traumas de face exigem uma abordagem agressiva, porém cuidadosa da via aérea, pois a entrega inadequada de sangue oxigenado ao cérebro e outras estruturas vitais é o fator que leva mais rapidamente à morte dos pacientes traumatizados. Uma via aérea protegida, desobstruída e com ventilação adequada são essenciais para evitar hipoxemia e tem prioridade sobre o gerenciamento de quase todas as outras condições (ATLS, 2018).

Em ambiente odontológico, as complicações estão mais frequentemente relacionadas à perda das vias aéreas superiores do paciente como nas reações as soluções anestésicas. Por isso, devem ser realizadas sempre observando-se as condições clínicas do paciente, bem como o reconhecimento por meio de monitoramento cuidadoso, observando a elevação do tórax do paciente, o uso de estetoscópio precordial e da oximetria de pulso sendo as chaves para a segurança nos atendimentos (Schwartz, 2018). Além disso, durante o tratamento clínico podemos observar um grande potencial dos objetos utilizados durante os procedimentos caírem na parte posterior da cavidade oral e, subsequentemente, dentro da faringe e ainda que uma situação de emergência potencialmente fatal não exista imediatamente, certos passos importantes devem ser realizados rapidamente para assegurar a remoção do objeto em um período de tempo razoável a fim de evitar sérias consequências ao paciente. Entretanto, existe a possibilidade de que tal objeto se fixar na laringe e obstruir completamente a traqueia. Desta forma, é imprescindível que o profissional esteja familiarizado com o correto manejo da desobstrução aguda das vias aéreas superiores (Malamed, 2015).

A obstrução das vias aéreas pode ser classificada como parcial ou completa, dependendo do mecanismo e causa. Uma obstrução completa causará hipóxia e parada cardíaca rapidamente, enquanto que a obstrução parcial pode ser mais enganosa no início, porém com a ventilação reduzida no paciente pode levar a hipercapnia, acidose respiratória e hipoxemia. Podemos classificar uma respiração com a presença de ruídos como uma via aérea parcialmente obstruída enquanto que a ausência completa de ruído, indica uma obstrução total (Yildirim, 2018).

Após revisão de literatura, este trabalho tem como objetivo apresentar as manobras de desobstrução e acesso às vias aéreas em situações de emergência odontológica, detalhando os procedimentos em ordem de prioridade, indicações e contraindicações. Podemos destacar as manobras de Heimlich, Jaw Thrust; Chin Lift, Intubação Endotraqueal, Cricotireiodostomia e Traqueostomia.

\section{Metodologia}

Realizou-se um estudo descritivo, do tipo revisão da literatura, onde buscamos elencar as principais ideias e conceitos sobre a temática exposta, ao qual permite-nos aprofundar, dentro de diversos autores e referenciais, sobre os discursos e principais temas abordados, fazendo um compilado que nos permite diversos olhares para um mesmo objeto de pesquisa (Pereira et al., 2018). A busca se deu de forma eletrônica pelas base de dados SciElo e Embase. Para isto, utilizou-se os descritores indexados e os operadores booleanos "Airway Obstruction" and "Emergency" or "Dentistry". Na triagem utilizando-se a interpretação e análise crítica do autor pela leitura dos títulos e resumos. Houve critérios de inclusão para produções em inglês e espanhol que foram publicados entre os anos de 2016 a 2020 e como critérios de exclusão os textos que não se adaptaram com a temática pesquisada. Mesmo com a buscas em artigos científicos, existem livros de literatura básica que abordam a temática pesquisada e por isso as obras relevantes também forma utilizadas com um total de 40 publicações científicas selecionadas (Figura 1). 
Figura 1. Fluxograma da metodologia de pesquisa e textos encontrados.

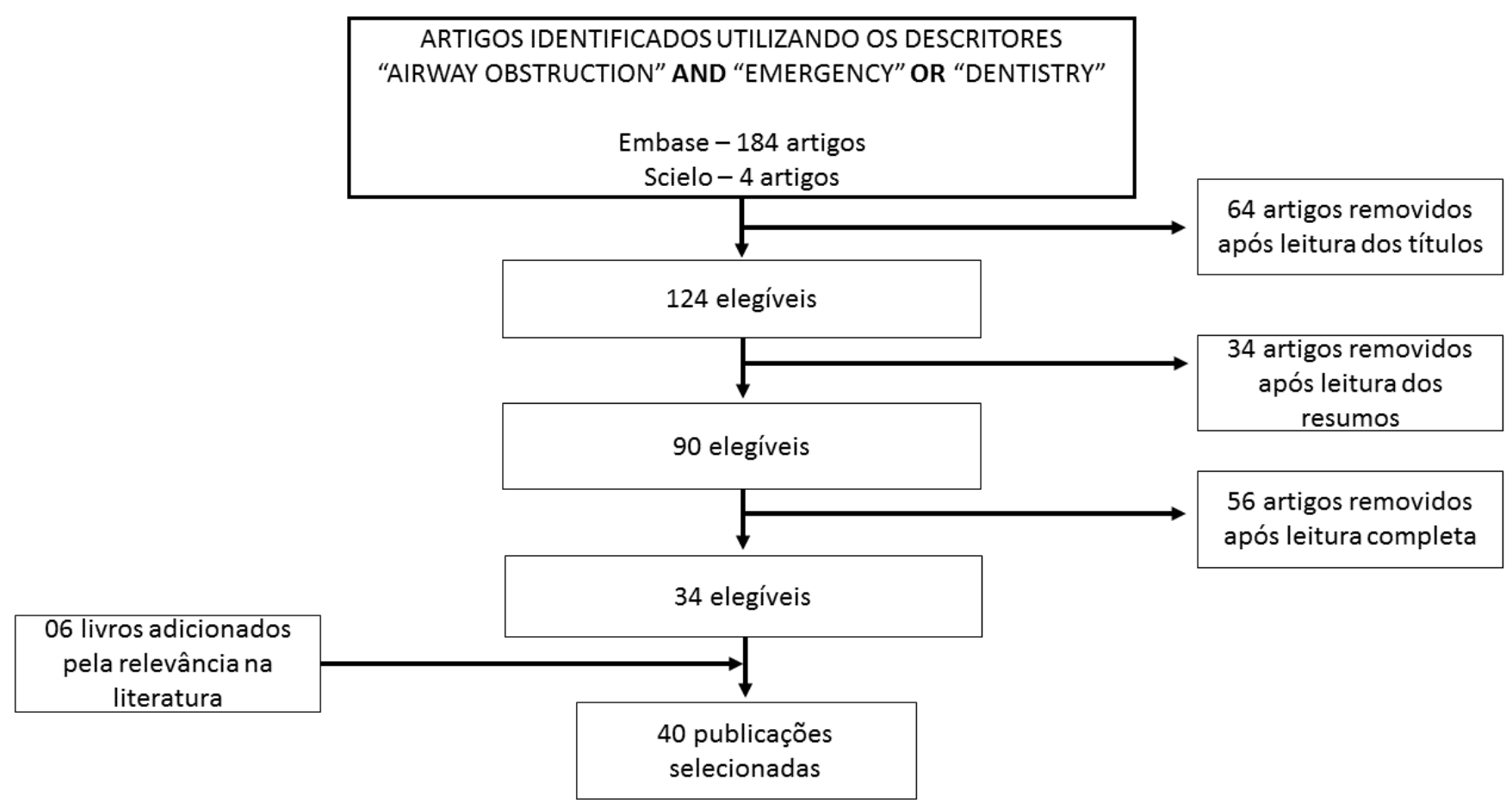

Fonte: Autores.

\section{Resultados e Discussão}

\subsection{Anatomia}

A compreensão da anatomia das vias aéreas e das estruturas anexas é essencial durante o atendimento das emergências, pois as abordagens são complexas e as complicações decorrentes do incorreto manejo das vias aéreas geralmente podem ser fatais (Menran, 2018).

\section{Cavidades nasais}

A anatomia da cavidade nasal é formada por uma abertura anterior denominada de narina e abre-se posteriormente na nasofaringe através das coanas (Moore, 2010). Possui duas cavidades paralelas que estão separadas por um septo osteocartilagíneo - o septo nasal - e são constituídas estruturalmente por teto, assoalho e as paredes lateral e medial em cada cavidade. Os cornetos nasais, geralmente em número de três (inferior, médio e superior), são saliências que se inserem na parede lateral da fossa nasal, forradas por uma mucosa denominada como membrana de Schneider, espessa e com abundante vascularização que desempenha papel relevante na fisiologia respiratória, promovendo a filtração, aquecimento e umedecimento do ar inspirado. As cavidades nasais, constituem, por isso, verdadeiras proteções das regiões vizinhas como cavidades paranasais e das vias aéreas inferiores (Hungria, 1991).

\section{Faringe}

A faringe é a parte superior do sistema digestório e respiratório, encontrando-se posterior às cavidades nasal e oral, estende-se, anteriormente da base do crânio até a margem inferior da cartilagem cricóidea e na margem inferior da sexta vértebra cervical, posteriormente. A parede posterior é plana e situa-se contra a lâmina pré-vertebral da fáscia cervical (Moore, 2010). 
Os músculos da faringe são compostos por três constritores circulares e três levantadores longitudinais. O suprimento arterial é derivado dos ramos da artéria carótida externa, a artéria faríngea ascendente, ramo palatino ascendente e ramo tonsilar da artéria facial, artéria maxilar e ramos dorsais da artéria lingual. As veias faríngeas começam em um plexo externo à faringe, recebem veias meníngeas e uma veia do canal pterigoideo, e geralmente terminam na veia jugular interna. Possuem comunicação com a nasofaringe, orofaringe e laringofaringe, respectivamente. Apresenta mucosa de revestimento contínua com aquela que reveste os tubos faríngeo timpânicos, cavidade nasal, cavidade oral e laringe (Gray, 1988).

\section{Laringe}

A laringe possui a função de proteger as vias aéreas durante a deglutição e da fonação através das cordas vocais. É delimitada superiormente pelo osso hioide e inferiormente pela cartilagem tireoide. É de grande relevância saber que, na composição da laringe temos cartilagens impares tireóidea, cricóidea e epiglótica e, as cartilagens pares aritenóide, cuneiforme, corniculada e tritícea. A cartilagem tireóidea é composta por duas lâminas de formato quadrilaterais que fundem-se formando a proeminência laríngea medialmente. Já a cartilagem tireoide é unida ântero-medialmente com a cartilagem cricóide através do ligamento cricotireóideo (Gray, 1988; Bailey \& Goldenberg, 2019). Essas diferentes cartilagens estão ligadas entre si por ligamentos e articulações. Toda inervação motora dos músculos da laringe é fornecida pelo nervo laríngeo inferior e superior. A vascularização arterial da laringe é garantida pelas artérias laríngeas (superior, média e inferior), ramos das tireóideas (Hungria, 1991).

\section{Traqueia}

A traqueia é uma estrutura tubular, cartilaginosa delimitada superiormente a altura de borda inferior da cartilagem cricóide e inferiormente até a Carina da traqueia. Possui entre 10 a $13 \mathrm{~cm}$ de comprimento, sendo mais curta nas mulheres e é composta de 18 a 22 anéis traqueais, com em média de $4 \mathrm{~cm}$ de altura, esses anéis são estruturas que se apresentam em forma de C com uma parede posterior membranosa. (Furlow \& Marthisen, 2018). A primeira cartilagem traqueal fica imediatamente abaixo da cartilagem cricóide, sendo o único anel cartilaginoso contínuo (Mehran,2018). É suprida pelos ramos das artérias tireóideas inferiores e ramos das artérias brônquicas, a drenagem da traqueia acontece pelas veias e terminam no plexo venoso tireóideo inferior (Gray, 1988).

\subsection{Manejo das vias aéreas}

\subsubsection{Manobras básicas}

\section{Manobra de Heimlich}

A manobra de Heimlich foi descrita pela primeira vez em 1974 como uma técnica de primeiros socorros para evitar asfixia por obstrução das vias aéreas. Para ser executada não precisa de instrumentos, podendo ser realizada por qualquer pessoa, o que demonstra a facilidade do procedimento. Pode-se observar que ainda no ano de 1975, nove meses depois da primeira descrição,162 pessoas tinham sido salvas por essa manobra (Heimlich, 1974). A base dessa técnica é a criação de uma tosse artificial, elevando vigorosamente o diafragma e forçando o ar dos pulmões. (Ichikawa et al., 2017). Por se tratar de um procedimento não cirúrgico, raramente apresenta complicações, porém ruptura de estômago é relatada na literatura como a mais comum. Todo paciente em que a manobra for realizada e apresentar alguma sintomatologia, precisa ser observado e incluir um exame físico completo para descartar possíveis complicações (Herman et al., 2018).

Quanto à epidemiologia podemos observar que a população a qual predominantemente realiza-se a manobra de Heimlich são idosos, por serem mais vulneráveis e estão altamente suscetíveis a asfixia devido a distúrbios 
neuromusculares, como alterações do sistema nervoso relacionado à idade, distrofia muscular, assim como os problemas dentários, que os colocam em situação de risco durante a deglutição. Por isso, é considerado o procedimento de escolha para o manejo inicial das vias aéreas obstruídas quando as técnicas de suporte básico de vida se mostram inadequadas (Ebrahimi et al., 2019).

Para prestar assistência à vítima consciente com obstrução grave das vias aéreas, o Cirurgião-Dentista deve pedir o consentimento para então pode atuar, salvando-se os casos de emergências, pacientes pediátricos e deficientes que o consentimento é tácito. A técnica consiste em posição estável em pé ou ajoelhado, na qual a pessoa que vai realiza-la deve se posicionar atrás da vítima com os braços ao redor da cintura. Em seguida, posicionar uma mão fechada contra o abdômen da vítima abaixo do apêndice xifoide e com a outra mão, segurar a mão fechada pressionando com um impulso rápido e forte para dentro e para cima, repetindo essas compressões até que o objeto seja expelido. Em pessoa inconsciente (não responsiva), deve-se colocar em posição supina, realizar a chamada do serviço médico e iniciar os protocolos de suporte básico à vida realizando-se 30 compressões torácicas e duas ventilações. Entre esse protocolo, deve-se abrir a boca do paciente e realizar a inspeção visual e se o objeto for visível,deverá ser removido com um fórceps de intubação de Magill, uma pinça de algodão ou com os dedos. Em pacientes gestante ou obesa, as compressões torácicas devem ser administradas no lugar das compressões abdominais (Malamed., 2015).

\section{Jaw Thrust}

Também conhecida como tração mandibular, essa manobra serve para anteriorizar o osso hioide e a língua para longe da parede faríngea (Sykes, 2019). É utilizada em pacientes inconscientes, pois a hipotonia da língua pode obstruir a hipofaringe. Para corrigir prontamente essa forma de obstrução, é indicado pelos profissionais de saúde utilizarem as manobras de Jaw Thrust ou Chin Lift (ATLS, 2018).

Inicialmente, em pacientes com suspeita de trauma na cabeça, pescoço ou face, a coluna cervical deve ser mantida em uma posição estável, uma vez que a manobra de tração mandibular permite que o profissional limpe as vias aéreas com pouco ou nenhum movimento da cabeça e da coluna cervical. Por isso, a técnica consiste em primeiro certificar-se da estabilidade da coluna cervical, em seguida deve-se colocar os polegares em cima do osso zigomático em ambos os lados da face e os dedos indicadores e médio no ângulo da mandíbula, tracionando-a para a frente (PHTLS, 2018).

A manobra de Jaw Thrust apresenta desvantagens em pacientes com limitações de abertura bucal uma vez que a realização da tração mandibular será mais difícil (Mohseni, 2017).

\section{Chin Lift}

Também conhecida como elevação do mento é uma manobra simples para abrir e manter uma via aérea pérvia, sendo indicada em pacientes inconscientes (Sykes, 2019). A técnica consiste em se posicionar de lado do paciente e colocar a palma de uma mão sobre a região frontal, empurrando suavemente para realizar uma hiperextensão da cabeça. Os dedos da outra mão do socorrista devem ser colocados sob a região de sínfise mandibular da vítima levantando o queixo para frente, pois como a língua tem alguns de seus músculos inseridos na mandíbula, ela é tracionada para anterior e para longe da parede posterior da laringe, estabelecendo assim uma via aérea patente. Essa manobra é contra indicada em paciente politraumatizado de face com possível lesão cervical, pois esse movimento pode aumentar os riscos de danos às vértebras e a medula espinhal da vítima (Tevatia,2016). 


\subsection{Manobras avançadas}

\section{Intubação Endotraqueal}

A intubação endotraqueal é considerada o padrão-ouro nas práticas de gerenciamento e controle das vias aéreas. É indicada em pacientes críticos com incapacidade de proteger às vias aéreas da aspiração de conteúdo gástrico e sanguíneo provenientes do trauma, pacientes com falha em outros procedimentos de manutenção das vias aéreas, pacientes com baixa oxigenação e parada cardiopulmonar (Parviz, 2019). O manejo no trauma geralmente ocorre sob a suposição de que o paciente apresenta lesão instável na coluna cervical até que se prove o contrário. Embora a incidência de lesões cervicais seja relativamente baixa, é um acesso contraindicado nessas situações, pois pode causar lesões secundárias na medula espinhal (Kovacs \& Sowers, 2018).

Assim como em todo manejo avançado do suporte de vida, para realização da técnica é recomendado possuir o equipamento adequado que consiste em: Laringoscópio com lâminas para adulto e criança,do tipo retas e curvas; Equipamento de aspiração com cateteres rígidos e flexíveis; Tubo endotraqueal com tamanho adulto e pediátrico; Fio guia metálico; Sonda guia do tipo flexível; Seringa do tipo Luerde $10 \mathrm{ml}$; Lubrificante hidrossolúvel; pinça de Magill; dispositivo de detecção de final de expiração para ETCO2; Capnografia por onda; Dispositivo para fixação do tubo endotraqueal; Baterias extras e lâmpadas reserva (PHTLS, 2018).

A decisão para realizar a intubação endotraqueal deve ser tomada após a avaliação da via aérea. É importante reconhecer se esta é considerada difícil ou não para poder decidir sobre a utilização desse acesso. A American Society of Anesthesiologists (2003) define uma intubação traqueal difícil como aquela que requer múltiplas tentativas, na presença ou ausência de patologia traqueal. Qualquer condição que resulte em uma diminuição do diâmetro endotraqueal como compressão extratraqueal por tumor, abcessos locais, hematomas; macroglossia, queixo retraído, corpos estranhos intratraqueal ou doenças traqueais, aumentarão a dificuldade de intubação (Sun et al., 2017). Por isso, o método LEMON é utilizado para avaliar a dificuldade envolvida no procedimento.

Critérios de diagnóstico LEMON:

L (Look) - Olhar externamente. Procurando características externas que dificultem a intubação ou ventilação.

E (Evaluate) - Avalie com a regra 3 - 3-2. Para realizar uma intubação é necessário realizar o ajuste entre o eixo boca, faringe e laringe, o máximo possível ao realizar o procedimento. A regra $3-3$ - 2 é usada para avaliar essa relação. Utilizando os dedos, irá se observar a distância entre os incisivos superiores e inferiores do paciente com a boca aberta; A distância entre o osso hioide e o basilar mandibular e a distância entre a altura da cartilagem tireoide e o assoalho bucal, nesta ordem. Caso não se atinja ao menos os números dessa regra, indicará uma exposição difícil à glote sob laringoscopia direta (Sun et al., 2017; Mshelia, Obgoli-nwasor \& Isamade, 2018).

M (Mallampati) - Classificação modificada de Mallampati. É outra ferramenta para visualizar a hipofaringe e avaliar a dificuldade de intubação das vias aéreas. É solicitado que o paciente, em posição neutra, abra a boca e projete a língua para fora. A classificação pode ser descrita da seguinte forma:

Classe I: Palato mole, fauces, úvula, pilares visíveis.

Classe II: Palato mole, fauces, úvula visível.

Classe III: Palato mole, base da úvula visível.Classe

IV: Palato mole não visível.

Sendo as classes I e II correspondentes a fácil intubação e as classes III e IV denotam intubação difícil (Sanyal, 2019).

O (Obstruction) - Obstrução. Os pacientes são avaliados sobre qualquer condição que possa causar obstrução das vias aéreas como corpos estranhos, tumores, abscessos, epiglote inflamada ou hematoma em expansão. 
N (Neck mobility) - Mobilidade do pescoço. Este é um requisito vital para o sucesso na intubação. A avaliação se dar solicitando ao paciente que em posição sentada que coloque o queixo sobre o peito e, em seguida, estender o pescoço para que olhe para o teto (Mshelia, Obgoli- nwasor \& Isamade, 2018).

Dentre os métodos de intubação utilizados em situações de emergência, a sequência rápida de intubação (SRI) representa 85\% das escolhas (Pallin et al., 2016). A técnica é indicada em pessoas que possuam função circulatória preservada e em pacientes que não fizeram jejum, evitando aspiração de conteúdo gástrico e em pacientes que não apresentem vias aéreas difíceis com contraindicação de uso de bloqueadores neuromusculares (Hinkelbein \& Kranke, 2018).

\section{Sequência rápida de intubação}

A sequência rápida de intubação (SRI) utiliza-se do mnemônico referidos como os sete "Ps" que são a sequência de sete passos para realização da técnica ao qual embora descrita como ações individuais, são uteis ao ensinar ou planejar a técnica, porém a maioria das intubações de emergência exigem que as várias etapas ocorram simultaneamente. Os sete Ps são: Preparação; Pré-oxigenação; Pré-intubação (otimização); Paralisia com indução; Posicionamento; Posicionamento com comprovação; Pós-intubação (Brown III, Sakles \& Mick, 2018).

A técnica inicia-se com a etapa da preparação que envolve a separação de todo os equipamentos e suprimentos necessários para realizar uma intubação de emergência, além disso, realiza-se a colocação de um cateter intravenoso e o monitoramento dos sinais vitais como a frequência cardíaca, respiração, pressão arterial, oximetria de pulso, e se possível a monitoração do ritmo cardíaco por capnografia. A pré-oxigenação deve ocorrer junto com a etapa anterior, de forma à $100 \%$ com a máscara com válvula e balão durante 3 a 5 minutos com o objetivo de permitir apneia sem queda da saturação de oxigênio do paciente. A fase da pré-intubação (otimização) é a etapa ao qual poderão ser administrados fluidos isotônicos e agente vasopressores, se o tempo permitir, para melhorar as condições fisiológicas como a pressão arterial, aumentando as opções farmacológicas para a SRI. Após isso, vem a fase da paralisia com indução, um agente de indução de ação rápida é administradoem bolus intravenoso em dose adequada para produzir perda imediata de consciência. É feito o posicionamento do paciente para a intubação que após 20 a 30 segundos estará induzido, apneico e flácido. Após 45 a 60 segundos é feita a inserção com do tubo endotraqueal e confirmação do posicionamento após a intubação. Por fim, a fase da pós-intubação é o manejo ao qual vai ser fixado o tubo e iniciará a ventilação mecânica. Em geral, se depois de três tentativas o acesso falhar, essa técnica deve ser desconsiderada e então realizar um acesso cirúrgico das vias aéreas (Ross \& Ellard, 2016; Brown III, Sakles \& Mick, 2018; PHTLS, 2018).

\subsection{Acessos cirúrgico das vias aéreas}

Uma via aérea cirúrgica (isto é, cricotireoidotomia ou traqueostomia) é indicada na presença de edema da glote, fratura da laringe, hemorragia orofaríngea grave que obstrui a via aérea ou incapacidade de colocar um tubo endotraqueal através das cordas vocais.

\section{Cricotireiodostomia}

A cricotireiodostomia é o procedimento realizado para fornecer oxigenação de emergência que impede lesão neurológica permanente secundária da hipóxia ou a morte (Frennessy, 2019).

Quando a realização das manobras não invasivas não tem êxito e uma intubação de emergência não é possível de ser realizada, torna-se necessário lançar mão dos acessos cirúrgicos. A cricotireiodostomia é indicada quando o paciente apresentar uma anatomia de difícil acesso, sangramento ou edema da região de orofaringe que oculte a visualização, obstrução das vias aéreas como por manobra de Heimlich, malsucedida, obstrução das vias aéreas por angioedema ou queimaduras por 
inalação e nos traumas Buco Maxilo Faciais. A cricotireiodostomia é preferível a uma traqueostomia para a maioria dos pacientes que necessitam de uma via aérea cirúrgica de emergência porque é mais fácil de executar, associada a um menor sangramento e requer menos tempo para realização (ATLS, 2018; Bribiesco, 2018).

Macêdo e colaboradores (2016), realizou uma revisão sistemática da literatura para avaliar as cricotireoidostomias em hospitais de emergência, nove estudos do tipo retrospectivos foram selecionados com um total de 316 procedimentos. O estudo mostrou que aproximadamente $90 \%$ das indicações de cricotirodostomia foram decorrentes de trauma. A escolha dessa manobra de emergência se dá pela simplicidade da técnica e por preservar as estruturas anatômicas da região. Posteriormente é recomendada a conversão para traqueostomia, pois uma das possíveis complicações à longo prazo é a ocorrência de estenose supra glótica.

A cricotireoidostomia cirúrgica é contraindicada em crianças menores de 12 anos de idade, por conta dos danos à cartilagem cricóide, que é a única cartilagem que possui arco completo, sendo suporte para a traqueia (ATLS, 2018). Para a realização do procedimento, é necessário a hiperextensão da cabeça do paciente e realizar a palpação de pontos anatômicos importantes como a proeminência laríngea e a cartilagem cricóide, para identificação do ligamento cricotireoideo que se localiza na depressão entre esses pontos (Heymans, 20016).

A manobra segue com a realização de uma incisão transversal através da membrana cricotireóidea. Com a utilização de uma alça para bisturi ou uma pinça hemostática do tipo curva, deve-se inserir na incisão e realizar uma volta de $90^{\circ}$ graus para ampliar o campo cirúrgico e um tubo endotraqueal ou de traqueostomia (preferencialmente de 5 a $7 \mathrm{~mm}$ de diâmetro) deve ser inserido para começar a ventilação (ATLS, 2018).

\section{Traqueostomia}

A traqueostomia é um procedimento utilizado para criar uma via aérea cirúrgica segura através da região anterior do pescoço. A realização de uma traqueostomia é indicada em situações de obstrução das vias aéreas superiores, controle de secreções excessivas, necessidade de ventilação prolongada e de proteção a longo prazo das vias aéreas. Geralmente é realizada entre o segundo e o terceiro anel traqueal, pois incisões mais altas aumentam o risco de lesão da laringe e incisões mais baixas aumentam o risco de decanulação e lesão dos vasos da região (Bomtempo \& Manning, 2019).

A técnica consiste na realização de uma incisão transversal ou vertical através da pele que é feita entre a borda inferior da cartilagem cricóide e a fúrcula esternal. O tamanho geralmente é em torno de $3 \mathrm{~cm}$, mas pode se estender de acordo com as necessidades anatômicas de cada indivíduo. Em seguida é feita a divulsão dos tecidos subcutâneos, músculo platisma e divisão do ístimo da tireoide com a retração lateral das estruturas, criando-se o acesso, através do qual um tubo de traqueostomia é colocado (Muscat, Bill \& Simo, 2017; Lipton et al, 2020).

Segundo Evans e Mccahon (2018) nos hospitais de emergências as traqueostomias cirúrgicas são feitas principalmente por Cirurgiões Otorrinolaringologistas (50\%) e Cirurgiões e Traumatologistas Buco Maxilo Faciais (CTBMF) (37\%). No Brasil, não possuímos Cirurgiões Otorrinolaringologistas nas emergências o que corrobora ainda mais para a importância do conhecimento da especialidade da CTBMF sobre a técnica. Em um contexto de "não consigo ventilar, não consigo intubar" durante a anestesia geral, a traqueostomia normalmente não é incluída como manobra de escolha para gerenciamento de emergência, a menos que a experiência do profissional e o equipamento necessário estejam imediatamente disponíveis para fazêlo de forma rápida e eficaz, dentro de um tempo limitado. As complicações operatórias mais comuns incluem deslocamento de estruturas, sangramento, pneumotórax ou criação de falso trajeto para inserção da cânula e infecções da ferida cirúrgica (Evans \& Mccahon, 2018; Kligerman,2020). 


\section{Conclusão}

O adequado manejo das vias aéreas nas emergências odontológicas é imprescindível por parte do Cirurgião-Dentista, pois a identificação precoce do comprometimento das vias aéreas e assegurar a manutenção desse sistema é o que reduzirá o potencial de danos nessas situações.

A manobra de Heimlich é eleita de escolha nas obstruções de vias aéreas superiores em pacientes conscientes. As manobras de Jaw Thrust e Chin Lift podem ser realizadas em pacientes que perderam a consciência, no entanto, sendo esta última contraindicada em pessoas que apresentem potencial de lesão na coluna cervical, como nos pacientes politraumatizados de face. A intubação endotraqueal é considerada o padrão-ouro nas práticas de gerenciamento e controle das vias aéreas definitiva, porém é um procedimento que requer a utilização de uma ampla medicação e equipamentos para sua realização. A cricotireiodostomia é a técnica cirúrgica de acesso que deve ser preconizada quando todas as outras manobras falharam por ser uma técnica de rápida execução, enquanto que a traqueostomia é um procedimento de caráter eletivo, pois demanda maior tempo e habilidades do profissional que irá realizar.

Por isso, cada vez mais se torna imperativo o conhecimento atualizado dessas manobras e acessos, além de treino dos profissionais da Odontologia diante das situações de emergência a nível ambulatorial e hospitalar para que se possam evitar sequelas ou até mesmo o óbito dos pacientes.

\section{Referências}

American Society of Anesthesiologists (2003). Practice guidelines of management of the difficult airway: an updated report. Anesthesiology, 118(2), 1269 1277 .

ATLS - Advanced Trauma Life Support (2018). American College of Surgeons, $10^{\circ}$ ed.

Bailey, D., Goldenberg, D. (2019). Surgical anatomy of the larynx. Operative techniques inotolaryngology - Head and neck surgery, $30(4), 232-236$.

Bribriesco, A., Patterson, G. A. (2018). Cricothyroid approach for emergency access to the airway.Thorac surg clin, 28(3), 435 - 440.

Brown III, C. A., Sakles, J. C., \& Mick, N. W. (2018). The Walls manual of emergency airwaymanagement. 5. ed. Philadelphia: Wohers Kluwer.

Ebrahimi, M., Mirhaghi, A. (2019). Heimlich Maneuver Complications: A Systematic Review. Eurasian J Emerg Med., 18(3), $157-165$.

Frennessy, P. et al. (2019). Emergency cricothyroidotomy: an observational study to estimate optimal incision position and length. British Journal of anaesthesia, 122(2), $263-268$.

Furlow, P. W., Mathisen, D. J. (2018). Surgical anatomy of the trachea. Ann cardiothorac Surg, 7(2), 255 - 260.

Gray, H., Goss, C. M. (1988). Anatomia. Guanabara Koogan, 29ed.

Heimlich, H. (1975). A life - saving maneuver to prevent food - choking. JAMA, 234(4), 398 -401.

Herman, A. et al. (2018). Heimlich maneuver - induced diaphragmatic rupture and hiatal hernia. The american journal of the medical sciences, $355(4), 13$.

Heymans, F. et al. (2016). Emergency cricothyrotomy performed by surgical airway - naive medicalpersonnel. Anesthesiology, $125(2), 295$ - 303.

Hungria, H. (1991). Otorrinolaringologia. Guanabara Koogan, Rio de janeiro, $6^{\circ}$ ed., 5 - 13.

Ichikawa, M. et al. (2017). Influence of body position during Heimlich maneuver to relieve subpharyngealobstruction: a manikin study. Acute Med Surg, 4(1), 418-425.

Kligerman, M. P. et al. (2020). Tracheostomy complications in the emergency de department: A nationalanalysis of 38,271 cases. ORL, 82(2), 1-8.

Kovacs, G., Sowers, N. (2018). Airway management in trauma. Emerg Med Clin N Am, 36(1), $61-84$.

Lynch, L., Crawley, S. M. (2018). Management of airway obstruction. British Journal of Anaesthesia, 18(2), 46-51.

Macêdo, M. B. et al. (2016). Emergency cricothyrotomy: temporary measure or definitive airway? A systematic review. Rev. Col. Bras. Cir., 43(6), 493499.

Malamed, S. F. (2015). Medical emergencies in the dental office. Elsevier, $7^{\circ}$ ed.

Mehran, R. J. Fundamental and practical aspects of airway anatomy. Thorac Surg Clin., 28(2),117-125. 
Mohseni, M. et al. (2017). Effect of Jaw Thrust and BURP Maneuvers on Glottis Visualization During C-MAC Video laryngoscopy. Ann Anesth Crit Care, 14462.

Moore, K. L. (2011). Anatomia orientada para a clínica. 6. ed. Rio de Janeiro: Guanabara Koogan.

Msheila, D. B., Ogboli-nwasor, E. O., \& Isamade, F. S. (2018). Use of the "L-E-M-O-N" score in predicting difficult intubation in Africans. Nigerian Journal of Basic and Clinical Sciences, 15(1), 17-23.

Nama, A. et al. (2020). Management of a Case of Airway Obstruction in the Emergency Department. Isr Med Assoc J, 22(3), $193-194$.

Pallin, D. J. et al. (2016). Techniques and trends, success rates, and adverse events in emergency departmentpediatric intubations: A report from the national emergency airway registry. Annals of emergency medicine, 67(5), 610-615.

Parviz, D. et al. (2019). Knowledge of pre-hospital emergency medical technician about endotracheal intubation: A manikin study. Journal of pharmaceutical research international, $31(6), 1-7$.

Pereira, A. S. et al. (2018). Metodologia da pesquisa científica. Santa Maria. Ed. UAB/NTE/UFSM.

PHTLS - Soporte vital de Trauma Prehospitalario. (2018). Jones \& Bartlett Publishers, $9^{\circ}$ ed.

Ross, W., Ellaed, L. (2016). Rapid sequence induction. Anaesthesia tutorial of week, 1-8.

Sanyal, R. et al. (2019). Comparison of airway assessment by mallampati classification and cormark andlehane grading in indian population. Indian Journal of Clinical Anaesthesia, 6(1), $140-142$.

Schuwartz, A. (2018). Airway management for the oral surgery patient. Oral Maxillofacial Surg Clin NAm, 30(2), $207-226$.

Sun, F. et al. (2017). Clinical consensus of emergency airway management. J Thorac Dis, 9(11), 4599 - 4606.

Sykes, P., Elliotte. L., Roew. M. (2019). Maintaining the airway. InnovAiT, 12(10), 561 - 568.

Tevatia, S. et al. (2016). Medical emergencies in dental practice: A review. World journal of pharmaceutical and life sciences, 2(4), 129 - 145.

Yildirim, E. (2018). Principles of urgent management of acute airway obstruction. Thoracic surgery clinics, 28(3), 415 - 428.

Mehran, R. (2018). Fundamental and practical aspects of airway anatomy from glottis to segmental bronchus. Thorac Surg Clin, 28(1), 117-125.

Bontempo, L. J., Manning, S. L. (2019). Tracheostomy emergencies. Emerg Med Clin N Am, 37(1), 109-119.

Lipton, G. et al. (2020). Multispecialty tracheostomy experience. Ann R Coll Surg Engl, 1-5.

Evans, S. W., Mccahon, R. A. (2018). Management of the airway in maxillofacial surgery: part 1. Br J Oral Maxillofac Surg, 56(6),463-468.

Muscat, K., Bille, A., Simo, R. A (2017). Guide to open surgical tracheostomy. Shanghai Chest, 1(4), 1-7.

Hinkelbein, J., Kranke, P. (2018). Rapid sequence induction. Anasthesiol Intensivmed Notfallmed Schmerzther, 53(1), 631-634. 